\title{
An Introduction to Developing Surveys for Pharmacy Practice Research
}

\author{
Lauren C Bresee
}

\section{INTRODUCTION}

A survey is defined as "any activity that collects information in an organised and methodical manner about characteristics of interest from some or all units of a population using well-defined concepts, methods and procedures, and compiles such information into a useful summary form." ${ }^{1}$ A survey can be a useful tool for pharmacy practice research, particularly for evaluating qualitative information. ${ }^{2}$ Although surveys are one of the most frequently used tools for health research, including pharmacy practice research, they are also sometimes misused or inaccurately designed, which limits the conclusions that can be drawn from their results. ${ }^{2}$ As Boynton and Greenhalgh state, "Anybody can write down a list of questions and photocopy it, but producing worthwhile and generalisable data from questionnaires needs careful planning and imaginative design." ${ }^{3}$

It is clear that in order to draw valid conclusions from survey results, we must ensure the following 2 conditions are met: first, that using a survey design to conduct the research of interest is justified, that is, other methods such as a retrospective chart review or administrative data review would not be more likely than a survey to produce valid results; and second, that the survey is carefully planned and implemented. ${ }^{1-6}$ The purpose of this article is to review the process for selecting, designing, and implementing a survey while minimizing the potential limitations associated with survey design. To clarify, this article focuses on sample surveys only, where data are collected for a sample of a larger population in order to draw conclusions about the population as a whole. ${ }^{1}$ This approach differs from a census survey, where data are collected from all members of the population of interest. ${ }^{1}$ Given the time and resources typically needed to conduct census surveys, their methods are not covered in this article. Table 1 presents definitions of common terms used throughout this article.

\section{BENEFITS AND LIMITATIONS ASSOCIATED WITH SURVEYS}

The use of surveys to conduct research is often appealing because of the potential to collect a large amount of data at little

\section{Table 1. Definitions of Terms ${ }^{1,3,6}$}

\begin{tabular}{ll} 
Term & \multicolumn{1}{c}{ Definition } \\
\hline Generalizability & $\begin{array}{l}\text { Applicability of the results to the overall } \\
\text { population of interest; also referred to as } \\
\text { "external validity" } \\
\text { List of questions administered to survey } \\
\text { participants } \\
\text { Questionnaire } \\
\text { Reliability }\end{array}$ \\
$\begin{array}{l}\text { Extent to which the questionnaire } \\
\text { produces repeatable findings; also known } \\
\text { as "reproducibility" }\end{array}$ \\
Response burden & $\begin{array}{l}\text { Time and effort required of a respondent } \\
\text { to complete a questionnaire; related to } \\
\text { length of the questionnaire and complexity } \\
\text { of the questions }\end{array}$ \\
Validity & $\begin{array}{l}\text { Extent to which the questionnaire } \\
\text { measures what it is intended to measure; } \\
\text { also referred to as "accuracy" }\end{array}$ \\
\hline
\end{tabular}

or no cost and the availability of multiple modes for collecting data, such as the Internet, telephone, in-person interviews, and regular mail. ${ }^{3,7}$ Surveys are also useful for assessing variables, such as attitudes, beliefs, and quality of life, that are not commonly available through other traditional sources of research data (such as patient charts and administrative databases). ${ }^{2,3}$ Also, data collection tends to be easier with survey research than with other research methods, because a standardized questionnaire, designed specifically for your research question and study objectives, is used. ${ }^{1,6}$ Lastly, previously validated surveys are available in the literature and can sometimes be used or adapted for subsequent survey studies. If you choose to use a previously validated survey for your study sample, you are assured that the questionnaire has been pretested and is measuring what it is intended to measure (i.e., the questionnaire is valid), and you can be confident in comparing your sample results with others in the published literature. ${ }^{3}$

Despite the benefits of surveys, we sometimes find reports based on poorly designed or poorly implemented survey designs in the literature. ${ }^{2-4}$ Surveys are associated with disadvantages that can limit conclusions, and you must consider potential sources of error when planning your survey in order to minimize these 
limitations. The errors associated with a survey can be divided into 2 groups: sampling errors and nonsampling errors. ${ }^{1}$ Table 2 lists the types of sampling and nonsampling errors that can occur with surveys, along with their potential causes. Strategies for avoiding these errors through survey design are reviewed later in this article.

\section{BEFORE CREATING A SURVEY PROTOCOL}

The first step in any research is developing your research question and study objectives (with relevant stakeholders, if appropriate), as reviewed in a previous article in the Research Primer series. ${ }^{8}$ Once you have established your research question and study objectives, you need to determine whether a survey is the most appropriate method for evaluating your study objectives. ${ }^{3,4}$ If your study objective is to assess the adequacy of blood pressure control in patients with hypertension in relation to the medications that patients are taking, obtaining blood pressure readings and a list of medications dispensed from the patients' medical records is likely to provide more detailed and useful information than conducting a survey. If you are interested in the patient's knowledge or beliefs regarding medication use for blood pressure control, a survey is likely the best method. It is important to note that your research question and study objectives dictate your survey methods, including the sample that should be surveyed, the mode of administering the survey, and the items to be included in your questionnaire, which further emphasizes the need for a clearly defined research question and study objectives. ${ }^{1,3,4}$

Once you have decided that a survey design is the most appropriate design for your study objective, your next step is to complete a literature review to identify previously published research that is relevant to your project. You can also use your literature search to determine whether a validated survey already exists that can be used to examine your study objectives. ${ }^{3}$ As mentioned above, using a previously validated survey questionnaire offers many advantages over designing your own questionnaire, including saving time and resources and allowing you to compare your study sample with previously published literature based on the same questionnaire. ${ }^{3}$

It must be noted, however, that prior publication of a particular questionnaire does not necessarily mean that it has been validated, nor does it mean that the questionnaire is reliable. ${ }^{3}$ Methods of determining the validity and reliability of a questionnaire are beyond the scope of this article, and you are encouraged to review detailed references on this topic., $69-11$ Examples of processes for validating questionnaires relating to pharmacy practice that have been implemented in Canada are also available. ${ }^{12,13}$

\section{STEPS IN CREATING A SURVEY PROTOCOL}

Let's assume that you have developed a clearly defined research question and one or more study objectives and you've determined that a survey is the most appropriate method for answering the research question. Even if you have found a validated survey questionnaire to use in your study, you will need to construct a survey protocol to ensure you have a clear plan for implementing the survey and to avoid the common errors associated with surveys. ${ }^{1}$ Statistics Canada conducts a significant number of surveys each year, including 18 currently active healthrelated surveys. ${ }^{14}$ In addition, despite the sample sizes of the surveys it conducts, Statistics Canada surveys often have impressive response rates, which illustrates the rigour of its survey designs. ${ }^{1}$ As a result, Statistics Canada is an excellent resource for creating and conducting surveys, and information from Statistics Canada's Survey Methods and Practices manual ${ }^{1}$ has been reviewed and summarized here as a guide to creating your survey protocol.

\section{Clearly Specify Your Target and Sample Populations of Interest}

From your research question and study objectives, you must first clearly define the target population that is to be studied. ${ }^{1}$ Your target population is "the population for which information

\section{Table 2. Sampling and Nonsampling Errors Associated with Surveys and Their Causes ${ }^{1}$}

\begin{tabular}{|c|c|c|}
\hline Type of Error & Definition & Causes \\
\hline Sampling error & $\begin{array}{l}\text { Error that arises when the survey sample is } \\
\text { not representative of the population to } \\
\text { be evaluated }\end{array}$ & Inappropriate choice of sampling frame \\
\hline \multicolumn{3}{|l|}{ Nonsampling errors } \\
\hline Measurement error & $\begin{array}{l}\text { Difference between the response recorded by } \\
\text { the individual and the true value }\end{array}$ & $\begin{array}{l}\text { Unclear questions; inadequate training of } \\
\text { interviewers; inappropriate mode of survey } \\
\text { administration; inaccurate translation (for } \\
\text { surveys administered in multiple languages) }\end{array}$ \\
\hline Processing error & Inaccurate coding of survey responses & $\begin{array}{l}\text { Lack of a data dictionary; inadequate training } \\
\text { of coder(s); use of multiple coders }\end{array}$ \\
\hline Nonresponse error & $\begin{array}{l}\text { Error caused by missing responses on some } \\
\text { of the questions in the questionnaire (partial } \\
\text { nonresponse) or the entire questionnaire } \\
\text { (total nonresponse) }\end{array}$ & $\begin{array}{l}\text { Questions about potentially sensitive topics; } \\
\text { inappropriate mode of survey administration } \\
\text { for the selected sample; poor design of } \\
\text { questionnaire; response burden (questionnaire } \\
\text { too long and/or too complex) }\end{array}$ \\
\hline
\end{tabular}


is desired". ${ }^{1}$ Questions that should be answered when clearly defining your target population include the following: What are the defining characteristics necessary for your sample to evaluate your study outcomes (e.g., disease states, age, education, use of certain medications, visits to your clinic)? What is the geographic location of your target population? (Are you including people from your hospital? Your city? Your province?) What is the time period under consideration?

Your sample population is the group of individuals to whom you will administer your survey questionnaire. ${ }^{1}$ In some cases, if the target population is small enough, your sample population may be the full target population. ${ }^{1}$ In many cases, however, time and resources are limited and you may therefore be able to include only a sample from your target population. If this is the case, it is important that your sample population be representative of the target population, to ensure that the survey results will be generalizable to your target population and to avoid sampling error. ${ }^{1}$ If you find that your sample is not representative of your target population, conclusions about the survey results can be applied only to the sample population. ${ }^{1}$

Calculating an appropriate sample size for your survey is complicated by a number of factors, including the desired precision of the estimate, the variability of your target population, the anticipated response rate, and how participants are identified (e.g., simple randomized sampling, systematic sampling, or more complex sampling techniques). ${ }^{1}$ Statistics Canada provides an excellent review of sample-size determination in its Survey Methods and Practices manual. ${ }^{1}$

\section{Select the Appropriate Survey Frame}

The survey frame refers to your methods for identifying and contacting your study sample. ${ }^{1}$ For example, if you wanted to survey members of the Canadian Society of Hospital Pharmacists (CSHP) about their attitudes toward conducting surveys for research, your survey frame could be the national list of members and their contact information (keeping in mind issues of privacy and access to personal information). If you wanted to survey homeless individuals regarding their access to medications, using the telephone book to randomly select individuals would be an inappropriate survey frame. Instead, approaching homeless shelters about conducting your survey would be a more effective way of identifying your survey frame. Within your sampling frame, you will want to ensure that you have identification information (full name or unique identifying number), contact information (address, phone number, e-mail address, as applicable to the target population), classification data (for example, if you specifically wanted to survey residency-trained CSHP members regarding their attitudes about conducting research surveys, you would need a way to identify those CSHP members who have residency training), and linkage data (if you plan to link with other data sources). ${ }^{1}$

\section{Develop Your Survey Questionnaire and Introductory Letter}

Researchers sometimes use the terms "survey" and "questionnaire" synonymously. Technically, though, the questionnaire is the sequence of questions that study participants answer, whereas the survey incorporates all steps of the research project, including designing and administering the questionnaire. ${ }^{1}$

If you have identified a previously validated questionnaire during your literature search, the step of questionnaire development will be partially complete, although you still need to create an introductory letter to accompany the questionnaire. If you have not identified a previously validated questionnaire, this is the point at which you construct your own survey questionnaire. Also important to include with your questionnaire is an introductory letter for potential study participants. ${ }^{1}$ The introductory letter should outline of the purpose of the survey and how the results of the questionnaire will be used; instructions on how to complete the questionnaire; information about confidentiality of the results, along with a statement that completing the survey is entirely voluntary and that the respondent can stop completing the questionnaire at any time; approximate length of time it will take to complete the questionnaire; relevant contacts for the study; and how to return the completed survey, if applicable. ${ }^{4}$

The following 3 hallmarks of a well-designed questionnaire will ensure that the results obtained are valid and that nonsampling errors are avoided' ${ }^{1}$ :

- efficient collection of data while avoiding errors and inconsistencies

- avoidance of response burden by ensuring that the survey is neither too long nor too complex

- minimization of the costs and resources associated with data collection

\section{Steps in Designing your Questionnaire}

In terms of designing the questionnaire, you should first consult with relevant stakeholders and data users to ensure that important information will be gathered. Specifically, what information do you need to collect from study participants to measure

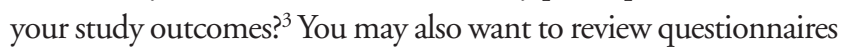
identified in your literature search that were only partially relevant to your study objective, because certain of their questions may be applicable to your study objectives. ${ }^{1}$ You will then draft your questions, pretest and revise them, and then finalize your questionnaire. ${ }^{1}$

\section{Types of Questions}

When drafting your questionnaire, you must balance the amount and granularity of information you would like to gather with the possibility of response burden. ${ }^{1,3,5}$ The 2 options for question design are open-ended questions and closed-ended questions. Closed-ended questions tend to minimize response 
burden and their responses are easier to analyze, but they may limit the detail of the information you would like to collect relative to open-ended questions. ${ }^{1,3}$ If you choose to include open-ended questions in your questionnaire, it is important to specify, before implementing the survey, how you will analyze the responses. ${ }^{1,3}$

In terms of closed-ended questions, some are intuitive and require minimal explanation (for example, asking the participant to state age in years, as of the date of questionnaire administration). Others, such as scaled questions, may require more detail to ensure you obtain a valid response. For example, if you are interested in gathering information on the amount of medication a person is using for breakthrough pain, the question "How often do you use medication for breakthrough pain?" with response options of "never", "sometimes", and "frequently" will not be useful unless you clearly define these options. ${ }^{3}$ For example, one respondent might interpret a frequency of one breakthrough dose per week as "sometimes", whereas another might interpret the same dosage as "frequently". To make the responses more informative, provide quantitative values within a meaningful range.

The 5-point Likert scale (strongly agree, agree, neutral OR neither agree nor disagree, disagree, strongly disagree) and similar scales are often used to measure respondents' strength of agreement or disagreement. There is debate about offering a midpoint ("neither agree nor disagree") on the Likert scale; excluding the midpoint option is called a forced choice, because the "neutral" or "neither agree nor disagree" option is not available. ${ }^{15}$ If the midpoint is excluded, participants may be more discriminating in their responses, but they may also become frustrated and record inaccurate responses because a neutral response is unavailable. ${ }^{15}$ As a result, if your survey includes questions with response options on a Likert scale, it is important to weigh the risks and benefits of including the neutral option as they relate to the question. If you are asking respondents about their agreement with a statement that is provided in the questionnaire, "neither agree nor disagree" may be a valid and informative response in relation to your study objectives.

Also, where necessary, be sure to specify the unit of measure within your questions. For example, if you are asking for the respondent's weight, you should also specify whether you want the weight in kilograms or in pounds.

Lastly, in addition to items relevant to your study objectives, it is important that the content of the survey include demographic information about the respondents, to allow you to compare your sample with the overall population of interest and thus to identify the potential generalizability of the results to your target population.

\section{Length of Your Questionnaire}

The length of your questionnaire is related to the likelihood of response burden - the longer the questionnaire, the greater the likelihood of response burden, which may result in either nonresponse or inaccurate responses. ${ }^{3}$ This problem must be balanced against the amount of data necessary to effectively evaluate your study outcomes. ${ }^{3}$ To minimize the length of your questionnaire, ask only questions that relate to your study objectives, and minimize (or eliminate) the use of repetitive questions. ${ }^{1,3}$ Although it is often appealing to collect as much data as possible, doing so should not be at the expense of limiting the validity of the responses.

\section{Appearance of Your Questionnaire}

Researchers occasionally forget to ensure that the appearance of the questionnaire does not discourage its completion. ${ }^{3}$ Items like font size and amount of white space can have a significant impact on response rates: if the font is too small or there are limited amounts of white space, survey participants may be discouraged from completing the questionnaire. ${ }^{3}$ It is also important to ensure that the appearance is specific to your target population. For example, some study groups may require larger font sizes on a printed questionnaire.

\section{Pretesting Your Questionnaire}

Your questionnaire should be pretested by a sample of individuals who are similar to your intended study sample. ${ }^{1}$ The purposes of pretesting are to ensure that instructions for completing the questionnaire are clear, to identify and revise confusing or ambiguous questions, and to estimate the time required to complete the survey and find out whether respondents find the survey too time-consuming, which could increase the risk for nonresponse error. ${ }^{1}$ If you revise your questionnaire after the first round of pretesting, you may want to pretest the updated version to ensure that the issues of concern have been resolved and that no new issues have been created.

\section{Data Collection}

Data collection is the process of gathering questionnaire responses from each survey participant. ${ }^{1}$ Central to data collection is identifying how you will collect your questionnaire data, that is, the mode for administering your survey. Common modes for administering a questionnaire include the Internet, telephone, regular mail, and in-person interviews. The common advantages and disadvantages of each mode of administration are listed in Table 3.

\section{Data Capture and Coding}

With some forms of data collection, the data can be captured in a format suitable for analysis at the time of questionnaire administration. If not, data capture is the process of entering your study data into statistical software that facilitates data analysis, such as Microsoft Excel or SPSS software. ${ }^{1}$ Data coding is the process of converting questionnaire responses into numeric codes 
This single copy is for your personal, non-commercial use only.

For permission to reprint multiple copies or to order presentation-ready copies for distribution, contact CJHP at cjhpedit@cshp.ca

Table 3. Advantages and Disadvantages of Commonly Used Modes of Survey Questionnaire Administration

\begin{tabular}{|c|c|c|}
\hline Mode of Administration & Advantages & Disadvantages \\
\hline Internet & $\begin{array}{l}\text { Less expensive and less time-consuming than } \\
\text { other modes; as a result, may allow for a larger } \\
\text { sample size relative to other modes; usually } \\
\text { anonymous, so potentially greater likelihood } \\
\text { of accurate responses, particularly for questions } \\
\text { about potentially sensitive topics }\end{array}$ & $\begin{array}{l}\text { May require valid e-mail addresses (if this is } \\
\text { how study participants will be informed of the } \\
\text { survey); requires that study participant have } \\
\text { access to a computer and be computer } \\
\text { literate; participant is unable to ask for } \\
\text { clarification of questions if confused }\end{array}$ \\
\hline Telephone & $\begin{array}{l}\text { Study participant is able to ask for clarification; } \\
\text { may yield higher response rate than mailed } \\
\text { or Internet-based surveys }\end{array}$ & $\begin{array}{l}\text { Requires valid telephone numbers to contact } \\
\text { participants; interviewer must be adequately } \\
\text { trained to administer the questionnaire; can } \\
\text { be time-consuming; potentially lower likelihood } \\
\text { of accurate responses, particularly for questions } \\
\text { about potentially sensitive topics }\end{array}$ \\
\hline In-person & $\begin{array}{l}\text { Study participant is able to ask for clarification; } \\
\text { may yield higher response rate than mailed } \\
\text { or Internet-based surveys }\end{array}$ & $\begin{array}{l}\text { Expensive and time-consuming; interviewer } \\
\text { must be adequately trained to administer the } \\
\text { questionnaire; potentially lower likelihood of } \\
\text { accurate responses, particularly for questions } \\
\text { about potentially sensitive topics }\end{array}$ \\
\hline$\overline{\text { Mail }}$ & $\begin{array}{l}\text { Anonymous, so potentially greater likelihood } \\
\text { of accurate responses, particularly for questions } \\
\text { about potentially sensitive topics; less time- } \\
\text { consuming than telephone or in-person }\end{array}$ & $\begin{array}{l}\text { Can be expensive, depending on sample size } \\
\text { of the study, number of survey reminders } \\
\text { mailed, and number of return postage-paid } \\
\text { envelopes provided to study participants; } \\
\text { administration requires valid mailing addresses }\end{array}$ \\
\hline
\end{tabular}

to facilitate analysis. ${ }^{1}$ When coding your data, it is helpful to create a corresponding data dictionary to record the meanings of the codes, particularly if multiple people will be imputing and coding the data. ${ }^{1}$ Your data dictionary should include a list of all the questions asked of participants, as well as the corresponding list of potential responses for each question. You can then use your data dictionary to keep track of the numeric responses recorded in the statistical software. For example, if you asked study participants whether they had been given a diagnosis of diabetes, and the possible responses were "Yes" and "No", the resulting data would typically be coded $0=$ No and $1=$ Yes; these numeric values would be reflected in your data dictionary.

\section{Data Analysis}

Data analysis is the process of summarizing your data to produce meaningful estimates based on your study objectives. ${ }^{1}$ Although a discussion of suitable methods for analyzing survey data is beyond the scope of this article, it is worthwhile to note the importance of clearly specifying the analytic plan, including the statistical tests that will be used to analyze your data, as part of your survey protocol, before initiating the survey. ${ }^{1}$ In addition, your analysis will vary depending on the type of questions included in your survey, such as open-ended questions or Likert-scale questions. . $^{1,3,16}$

\section{Dissemination and Reporting of Results}

Discussion of knowledge translation and mobilization strategies is similarly beyond the scope of this paper, but again it is worthwhile to emphasize the importance of clearly specifying a plan for disseminating and reporting your results. ${ }^{1,17}$ Your dissemination plan should be developed with the relevant stakeholders who are interested in your study results.

\section{ETHICS AND INSTITUTIONAL APPROVAL CONSIDERATIONS}

You have now created a survey protocol and are ready to get started. As highlighted in a previous article in the Research Primer series, ${ }^{18}$ research projects, including survey studies, require ethics approval from a research ethics board before the project can begin. In addition, if you are conducting the survey within one or multiple institutions, institutional approval may also be required. Although some surveys may appear innocuous, given the sample to be surveyed and the study objectives, it is always best to check with your local research ethics board, as well as the relevant institutional review boards, to find out whether approval is required. ${ }^{18}$

\section{CONCLUSIONS}

A survey can be an excellent method of collecting detailed qualitative data for a pharmacy practice research study. ${ }^{4}$ Surveys are also associated with significant disadvantages that must be considered when such a study is designed. ${ }^{1}$ Creating a concise research question and study objectives, confirming that a survey is the most appropriate study design to achieve your study objectives, and creating a detailed survey protocol before initiating your study are vital steps to minimize or avoid the common errors associated with survey designs. ${ }^{1,3,4}$ 
References

1. Survey methods and practices. Catalogue no. 12-587-X. Ottawa (ON): Statistics Canada; 2010 [cited 2014 May 2]. Available from: www.statcan. gc.ca/pub/12-587-x/12-587-x2003001-eng.pdf

2. Desselle SP. Construction, implementation, and analysis of summated rating attitude scales. Am J Pharm Educ. 2005;69(5):Article 97.

3. Boynton PM, Greenhalgh T. Selecting, designing, and implementing your questionnaire. BMJ. 2004;328(7451):1312-5.

4. Reierson Draugalis J, Coons SJ, Plaza CM. Best practices for survey research reports: a synopsis for authors and reviewers. Am J Pharm Educ. 2008; 72(1):Article 11.

5. Smith F. Health services research methods in pharmacy. Survey research: (1) Design, samples and response. Int J Pharm Pract. 1997;5(3):152-66.

6. Smith F. Health services research methods in pharmacy. Survey research: (2) Survey instruments, reliability and validity. Int J Pharm Pract. 1997; 5(4):216-26.

7. Couper MP. The future of modes of data collection. Public Opin Q. 2011; 75(5):889-908.

8. Tully MP. Research: articulating questions, generating hypotheses, and choosing study designs. Can J Hosp Pharm. 2014;67(1):31-4.

9. Cook DA, Beckman TJ. Current concepts in validity and reliability for psychometric instruments: theory and application. Am J Med. 2006;119(2): 166.e7-16.

10. Sullivan GM. A primer on the validity of assessment instruments [editorial]. J Grad Med Educ. 2011;3(2):119-20. Erratum: J Grad Med Educ. 2011; 3(3):446.

11. Kimberlin CL, Winterstein AG. Validity and reliability of measurement instruments used in research. Am J Health Syst Pharm. 2008;65(23):2276-84.

12. Guirguis LM, Chewning BA. Development of a measure to assess pharmacy students' beliefs about monitoring chronic diseases. Res Social Adm Pharm. 2008;4(4):402-16.

13. Guirguis L, Cooney D, Dolovich L, Eberhart G, Hughes C, Makowsky M, et al. Exploring pharmacists' understanding and adoption of prescribing in 2 Canadian jurisdictions: design and rationale for a mixed-methods approach. Can Pharm J. 2011;144(5):240-4.

14. Complete list of surveys and statistical programs. Ottawa (ON): Statistics Canada; 2014 [cited 2014 May 2]. Available from: http://www23. statcan.gc.ca/imdb/pIX.pl?Function=getThemeSV\&PItem_Id=97413\&PC E_Id=412\&PCE_Start=01010001\&CItem_Id=97413\&CCE_Id=412\&C CE_Start $=01010001 \&$ lang $=$ en

15. Rattray J, Jones MC. Essential elements of questionnaire design and development. J Clin Nurs. 2007;16(2):234-43.

16. Carifio J, Perla RJ. Ten common misunderstandings, misconceptions, persistent myths and urban legends about Likert scales and Likert response formats and their antidotes. J Soc Sci. 2007;3(3):106-16. Also available from: http://thescipub.com/abstract/10.3844/jssp.2007.106.116

17. Boynton PM. Administering, analysing, and reporting your questionnaire. BMJ. 2004;328(7452):1372-75.

18. Loewen P. Ethical issues in pharmacy practice research: an introductory guide. Can J Hosp Pharm. 2014;67(2):133-7.
Lauren C Bresee, BScPharm, ACPR, MSc, PhD, is with the Pharmacy, Alberta Health Services, Calgary, Alberta.

\section{Competing interests: None declared}

\section{Address correspondence to:}

Dr Lauren C Bresee

Pharmacy, Alberta Health Services

Room 302, South Tower

Foothills Medical Centre

Calgary AB T2N 2T9

e-mail: Lauren.Bresee@albertahealthservices.ca

This article is the fifth in the CJHP Research Primer Series, an initiative of the CJHP Editorial Board and the CSHP Research Committee. The planned 2-year series is intended to appeal to relatively inexperienced researchers, with the goal of building research capacity among practising pharmacists. The articles, presenting simple but rigorous guidance to encourage and support novice researchers, are being solicited from authors with appropriate expertise.

Previous article in this series:

Bond CM. The research jigsaw: how to get started. Can J Hosp Pharm. 2014;67(1):28-30.

Tully MP. Research: articulating questions, generating hypotheses, and choosing study designs. Can J Hosp Pharm. 2014;67(1):31-4.

Loewen $P$. Ethical issues in pharmacy practice research: an introductory guide. Can J Hosp Pharm. 2014;67(2):133-7.

Tsuyuki RT. Designing pharmacy practice research trials. Can J Hosp Pharm. 2014;67(3):226-9. 